\title{
Respiratory morbidity from lymphocytic interstitial pneumonitis (LIP) in vertically acquired HIV infection
}

\author{
M Sharland, D M Gibb, F Holland
}

\begin{abstract}
The aim of the study was to define the respiratory morbidity caused by lymphocytic interstitial pneumonitis (LIP) in children with vertically acquired HIV infection. A retrospective case note review was performed on 95 children attending three London hospitals. Clinical and radiological evidence of LIP, acute lower respiratory tract infections, and chronic lung disease was obtained using a structured protocol. A diagnosis of LIP had been made in $33 \%$, and an acute admission due to acute lower respiratory tract infection had occurred in $42 \%$ of all children (despite 99\% taking regular cotrimoxazole prophylaxis). Admission rates because of acute lower respiratory tract infection were significantly higher in the LIP group (0.38 admissions/child year) than in the non-LIP group $(0.17$ admissions/child year $)(p=0.0002)$. Encapsulated bacteria (Streptococcus pneumoniae, Haemophilus influenzae) were most frequently isolated. Improved methods of prevention of acute lower respiratory tract infection may help to reduce the severe respiratory morbidity seen in children with LIP and HIV infection.

(Arch Dis Child 1997;76:334-336)
\end{abstract}

Keywords: lymphocytic interstitial pneumonitis; HIV

Lymphocytic interstitial pneumonitis (LIP) was first described in association with autoimmune disease in adults. ${ }^{1}$ Histologically there is a diffuse lymphocytic infiltration of the interalveolar septa with proliferation of the bronchus associated lymphoid tissue (BALT). The association of LIP with adult and paediatric HIV infection was demonstrated on lung biopsies performed on individuals presenting with persistent bilateral reticulonodular shadowing on chest $x$ ray. ${ }^{23}$ Severe bacterial infections, especially of the lower respiratory tract, have also been recognised as a major problem in HIV infected children. ${ }^{45}$ The aim of this study was to determine the association between LIP, acute lower respiratory tract infections, and chronic lung disease in children with vertically acquired HIV infection attending three major hospitals in London.

Dr Mike Sharland, Paediatric Infectious Disease Unit, 5th Floor, Lanesborough Wing, St George's Hospital,

London SW17 0QT.

Accepted 31 December 1996

\section{Methods}

Children with vertically acquired HIV-1 infection who had received their clinical care from the paediatric infectious diseases units at $\mathrm{St}$ George's Hospital, St Mary's Hospital, or Great Ormond Street Hospital for Children were identified through the UK national study of HIV infection in children. The case notes were reviewed by a single investigator (MS) using a standard protocol. Children presenting with Pneumocystis carinii pneumonia were excluded from the study. Details extracted included dates of diagnoses of LIP and parotitis, details of hospital admissions with acute lower respiratory tract infection, and evidence of chronic lung disease. The following definitions were used:

Lymphocytic interstitial pneumonitis was defined as either confirmatory pathology on lung biopsy or a typical radiological appearance of bilateral diffuse reticulonodular shadowing seen on chest $x$ ray for over two months and unresponsive to antimicrobial treatment (fig 1). ${ }^{6}$ Parotid enlargement (bilateral and persisting for more than three months) was noted.

Acute lower respiratory tract infection was defined in a child requiring admission to hospital with the presence of at least four of the following five signs: fever greater than $38^{\circ} \mathrm{C}$; tachypnoea greater than 40 breaths/minute; new clinical chest signs; new radiological signs on chest $x$ ray; and prompt clinical response to antibiotic treatment. Length of hospital stay, and all positive cultures were noted.

Chronic lung disease was defined as the presence of a chronic cough (symptoms persisting for more than three months) plus chronic chest signs (persisting for more than three months), with or without clubbing of the fingernails. Comparison between the children in the LIP and non-LIP groups was made using the $\chi^{2}$ test. Rates of hospital admission (first admissions and recurrent admissions) were compared in the two groups using the normal approximation to the binomial distribution. ${ }^{7}$

\section{Results}

The case notes of 114 vertically HIV infected children who had received their main clinical care from one of the three hospitals were reviewed, covering the period 1984 to 1994 . Nineteen children had presented with Pneumocystis carinii pneumonia and were excluded from the analysis. Of the remaining 95 children, 43 were male, and 52 female. Seventy four per cent were black African, 13\% white, $10 \%$ mixed, and 3\% other. Fifty five (58\%) were born in the UK, but only 19 were followed up prospectively from birth. Of the children 


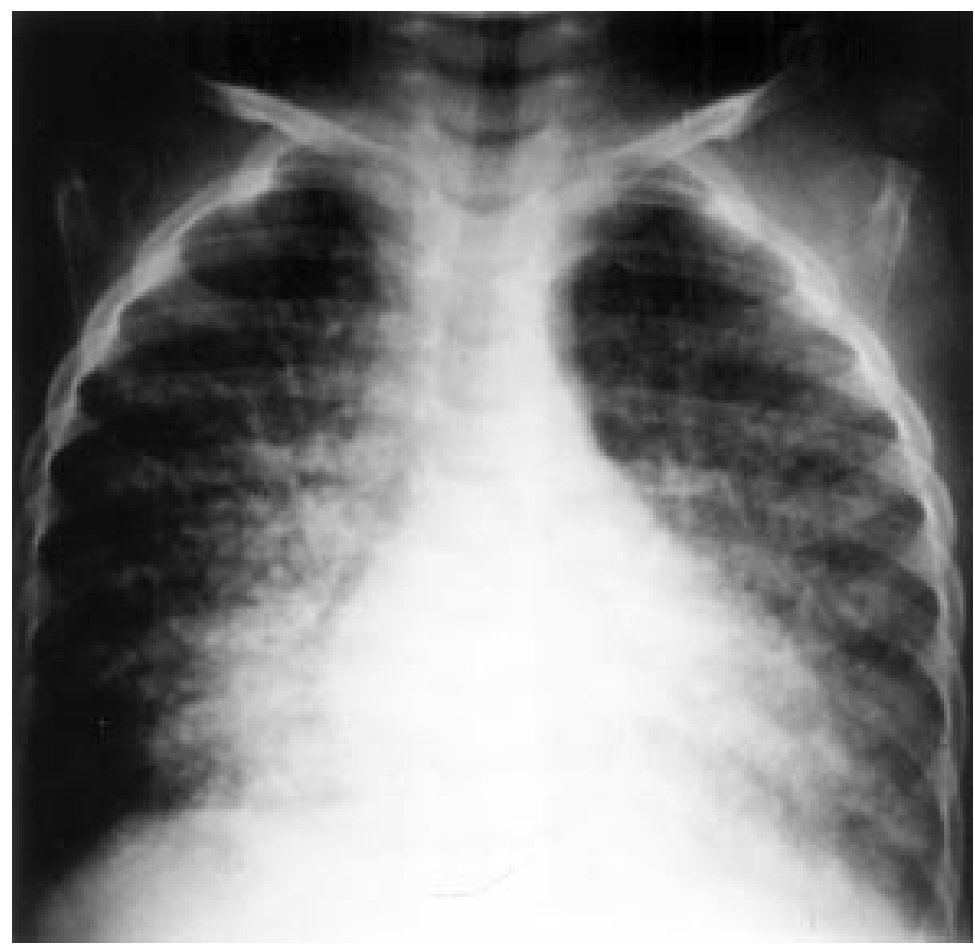

Figure 1 The chest $x$ ray of lymphocytic interstitial pneumonitis in a 5 year old girl with $H I V-1$ showing the bilateral reticulonodular shadowing.

born abroad the median age of arrival in the UK was 2 years (range 0 to 8 years). Thirteen of the 95 children had died. The median age of the 82 surviving children was 4.7 years (range 1.3 to 15.7 years).

LYMPHOCYTIC INTERSTITIAL PNEUMONITIS

A diagnosis of LIP had been made in 31 children $(33 \%)$, of whom 19 were born in the UK. Only five children had this diagnosis confirmed on open lung biopsy. Age at diagnosis and follow up time since first presentation were similar among children with and without LIP (table 1). Among the 19 children born in the United Kingdom, the median age of LIP diagnosis was 1.8 years (range 0.4 to 5.1 years). LIP was diagnosed in 15 of the 39 black African children born in the UK, compared with four of the 16 white or 'other' children $\left(\chi^{2}\right.$ $=0.4, \mathrm{p}>0.5)$. Parotid enlargement (defined as bilateral and persistent for more than three months) was significantly more common in children in the LIP group $(68 \%)$ than in the non-LIP group $(3 \%)\left(\chi^{2}=45.5, \mathrm{p}<0.001\right)$. A chronic cough which was present in $32 \%$ of children overall and lasted a median of three years was also noted significantly more often in the LIP group $\left(\chi^{2}=13.2, \mathrm{p}<0.001\right)$ (table 1$)$.

ACUTE LOWER RESPIRATORY TRACT INFECTIONS Over the study period of 276 patient years of follow up, 40 children (42\%) required 68 admissions for acute lower respiratory tract infections comprising a total of 700 hospital bed days. The admission rate for first and recurrent admission per child year of follow up was 0.38 in children with LIP compared with 0.17 among children without LIP ( $\mathrm{z}$ score 3.73, $\mathrm{p}=0.0002$ : table 1). Although the median length of admission was similar for the LIP and non-LIP groups, multiple admissions were more frequent in the LIP group. A positive culture was documented from blood culture, sputum, nasopharyngeal aspirate, or on bronchoalveolar lavage during only 24 $(35 \%)$ of the 68 admissions with acute lower respiratory tract infection. A single organism was isolated in 15 episodes, Streptococcus pneumoniae being the commonest isolate (table 2). Dual pathology was found in nine cases, cytomegalovirus being the most common second isolate. No significant differences were seen between the LIP and non-LIP groups. Of the 95 children, all but one had been recorded as taking regular co-trimoxazole as prophylaxis against pneumocystis pneumonia. Intravenous immunoglobulin had been given to $11 / 95$ children $(12 \%)$ at some stage of the illness, while $32 / 95$ (34\%) had taken zidovudine. Fifteen children $(16 \%)$ had received bronchodilators, and prolonged courses of steroids had been used in three children, all with severe LIP. During admissions for acute lower respiratory tract infection, intravenous antibiotics were given during $63 / 68$ (93\%) of episodes, but ventilation was only required in only $4 / 68$ episodes $(6 \%)$.

\section{Discussion}

Lymphocytic interstitial pneumonitis as a manifestation of paediatric HIV disease oc-

Table 1 Respiratory morbidity by lymphocytic interstitial pneumonitis (LIP) diagnosis

\begin{tabular}{|c|c|c|c|c|}
\hline & $L I P$ & No LIP & Total & $\begin{array}{l}p \text { Value } \\
\text { (LIP v non LIP } \\
\text { groups) }\end{array}$ \\
\hline No of children & $31(33 \%)$ & $64(67 \%)$ & 95 & \\
\hline Born in the UK & 19 & 36 & 55 & \\
\hline Ethnicity (\% black African among those born in the UK) & 15 & 24 & 39 & $\mathrm{p}=0.5$ \\
\hline Sex & $18 \mathrm{~F}: 13 \mathrm{M}$ & $33 \mathrm{~F}: 31 \mathrm{M}$ & $51 \mathrm{~F}: 44 \mathrm{M}$ & \\
\hline Median follow up (child years) & 3.3 & 2.7 & 2.9 & \\
\hline Median age (range) years & $2.3(0.2$ to 8.7$)$ & $3.1(0.3$ to 8.4$)$ & & \\
\hline Parotid enlargement & $21(68 \%)$ & $2(3 \%)$ & $23(24 \%)$ & $\mathrm{p}<0.001$ \\
\hline Chronic cough & $18(58 \%)$ & $12(19 \%)$ & $30(32 \%)$ & $\mathrm{p}<0.001$ \\
\hline Chest signs & $6(19 \%)$ & $3(5 \%)$ & $9(9.5 \%)$ & $\mathrm{p}=0.1$ \\
\hline Clubbing of the nails & $5(16 \%)$ & $2(3 \%)$ & $7(7 \%)$ & $\mathrm{p}=0.1$ \\
\hline ALRTI rate (admissions/child year) & 0.38 & 0.17 & 0.25 & $\mathrm{p}<0.0002$ \\
\hline \multicolumn{5}{|l|}{ Admissions for ALRTI : } \\
\hline None & $14(45 \%)$ & $41(64 \%)$ & $55(58 \%)$ & \\
\hline $1-2$ & $11(35 \%)$ & $23(36 \%)$ & $34(36 \%)$ & \\
\hline $3-6$ & $6(20 \%)$ & 0 & $6(6 \%)$ & \\
\hline
\end{tabular}

ALRTI $=$ acute lower respiratory tract infection. 
Table 2 Positive microbial cultures detected during 24 of the 68 episodes of acute lower respiratory tract infection, and their site of origin. In 15 children only one organism was detected; in nine children, two organisms were detected

\begin{tabular}{lll}
\hline Organism & Positive cultures & Site \\
\hline Haemophilus influenzae & 5 & Sputum (2) \\
Streptococcus pneumoniae & 9 & Bputum (2) \\
& & BAL (3) \\
Staphylococcus aureus & & Blood cultures (4) \\
Mycobacterium avium intracellulare & 1 & BAL (1) \\
Parainfluenza & 1 & BAL (1) \\
Influenza & 3 & BAL (1) \\
Adenovirus & 2 & NPA (1) \\
Respiratory syncytial virus & 2 & BPL (1) \\
Cytomegalovirus & 4 & NPA (3) \\
& & BAL (1) \\
& 6 & BAL (4) \\
\hline
\end{tabular}

$\mathrm{BAL}=$ bronchoalveolar lavage $\mathrm{NPA}=$ nasopharyngeal aspirate.

curred in nearly one third of children in our study, a similar rate to that reported from North America. ${ }^{2}$ LIP was originally an AIDS defining diagnosis in the 1987 CDC classification of AIDS in children. In the revised classification of paediatric HIV disease, ${ }^{8}$ it was recognised that children with LIP as a first AIDS indicator disease had an improved survival compared with other AIDS indicator diseases, ${ }^{6}$ and LIP has been redefined as a stage $\mathrm{B}$ disease indicator. However, this study confirms that LIP is associated with considerable respiratory morbidity. Although the overall rate of acute lower respiratory tract infection in this study ( 0.25 episodes/child/year of follow up) is the same as that previously reported in HIV infected children, ${ }^{5}$ the incidence of such infections in children with LIP was twice that observed in the children with no LIP. The number of children with LIP who had recurrent admission for acute lower respiratory tract infection was particularly striking. The incidence of acute lower respiratory tract infections in children with LIP is approximately 10-fold higher than that reported in an American community based study of non-HIV infected children. ${ }^{9}$ This study provides evidence of the considerable chronic respiratory morbidity associated with LIP. Some children develop chronic lung damage and bronchiectasis, with a clinical picture similar to cystic fibrosis. While bronchodilators may be of some benefit, other specific treatments have not been systematically studied in LIP. Rubinstein et al have reported improved oxygenation in a small number of children with severe LIP after starting steroids, ${ }^{10}$ but no controlled trials of steroid treatment have been carried out.

The aetiology of LIP remains uncertain. The principal hypotheses suggest that LIP represents an abnormal lymphoproliferative re- sponse, either to HIV alone, or due to superinfection with another virus, the most likely candidate being the Epstein-Barr virus. ${ }^{11}{ }^{12}$ In the majority of children, a presumptive diagnosis of LIP is now made on radiological and clinical grounds. Lung biopsy had been performed in only five children in this study, at a time when there was little experience of LIP in HIV infected children. Parotid enlargement is confirmed as a useful marker for the presence of LIP in paediatric HIV infection.

Both paediatricians and parents need to be aware of the increased risk of pneumonia in children with LIP, and acute infections should be promptly treated with systemic antibiotics and physiotherapy. An improved understanding of the viral aetiology and immunopathology of LIP is required. Development of a scoring system would facilitate multicentre trials. The most urgent management strategies that need to be evaluated are treatment trials of inhaled or oral steroids, combined with the use of long term continuous antibiotic prophylaxis against encapsulated bacteria.

We thank Drs J Evans, S Walters, and V Novelli for their help during data collection. The National Study of HIV in Children is supported by AIDS Education and Research Trust (AVERT), and the Department of Health. Dr Diana Gibb is supported by a MRC clinical senior fellowship.

1 Carrington C, Liebow A. Lymphocytic interstitial pneumonitis [abst]. Am F Pathol 1966;42:36a.

2 Pitt J. Lymphocytic interstitial pneumonia. Pediatr Clin North Am 1991;38:89-95.

3 Joshi VV, Oleske JM. Pulmonary lesions in children with the acquired immunodeficiency syndrome: a re-appraisal based on data in additional cases and follow-up study of based on data in additional cases and follow-up study
previously reported cases. Hum Pathol 1986;17:641-2.

4 Krasinski K, Borkowsky W, Bonk S, et al. Bacterial Krasinski K, Borkowsky W, Bonk S, et al. Bacterial
infections in human immunodeficiency virus infected children. Pediatr Infect Dis f 1988;7:323-8.

5 Mofenson LM, Moya J. Intravenous immune globulin for the prevention of infection in children with symptomatic human immunodeficiency virus infection. Pediatr Res 1993;33 (suppl):S80-9.

6 Connor EM, Andiman WA. Lymphoid interstitial pneumonitis in pediatric AIDS. In: Pizzo PA, Wilfert CM, eds. The challenge of HIV infection in infants, children, and adolescents. 2nd Ed. Baltimore: Williams and Wilkins, 1994:467-81.

7 Rothman KJ. Modern epidemiology. London: Little, Brown, and Company, 1986;156.

8 Revised classification system for human immunodeficiency virus infection in children less than 13 years of age. MMWR 1994;43:RR12:1-10.

9 Denny FW, Clyde WA. Acute lower respiratory tract infections in nonhospitalised children. F Pediatr 1986;108: 635-46.

10 Rubinstein A, Bernstein LJ, Charyton M, et al. Corticosteroid treatment for pulmonary lymphoid hyperplasia in chiloid treatment for pulmonary lymphoid hyperplasia in chil-
dren with acquired immunodeficiency syndrome. Pediatr dren with acquired im

11 Andiman WA, Eastman R, Martin K, et al. Opportunistic lymphoproliferations associated with Epstein-Barr viral DNA in infants and children with AIDS. Lancet 1985;ii: 1390-3.

12 Katz BZ, Berkman AB, Shapiro ED. Serologic evidence of active Epstein-Barr virus infection in Epstein-Barr virus associated lymphoproliferative disorders of children with acquired immunodeficiency syndrome. $\mathcal{F}$ Pediatr 1992;120: $228-32$ 